\section{Aplicação de atributos geométricos para mapeamento de descontinuidades: Exemplos das Bacia de Campos, Neuquén e Potiguar}

José Eduardo M. Lira (Petrobras), Rogério de A. Santos (Petrobras), Frank C. Bulhões (Petrobras), Gleidson D. Ferreira (Petrobras), Arnaldo Tanaka (Petrobras), Julio G. Carvalho (Petrobras), Adriano Barreto (PETROBRAS)

Copyright 2019, SBGf - Sociedade Brasileira de Geofísica

This paper was prepared for presentation during the $16^{\text {th }}$ International Congress of the Brazilian Geophysical Society held in Rio de Janeiro, Brazil, 19-22 August 2019.

Contents of this paper were reviewed by the Technical Committee of the $16^{\text {th }}$ International Congress of the Brazilian Geophysical Society and do not necessarily represent any position of the SBGf, its officers or members. Electronic reproduction or represent any position of the SBG, its officers or members. Electronic reproduction or of the Brazilian Geophysical Society is prohibited.

\section{Abstract}

Fault and fracture detection is one of the most challenging task for oil exploration. These structures are the preferable pathways for the oil and gas to migrate through the reservoir rocks in low permeability reservoirs. Geometrical attributes are very useful in detecting and mapping fractured areas. The Minimal Similarity accumulation(MSA) attribute was successfully applied to onshore and offshore data in Marizeiro, Potiguar Basin, Marlim Leste, Campos Basin, both in Brazil, Neuquén basin, Argentina. The algorithm uses a neural network, along with a dimensionality reduction technique to speed up the process, to enhance fault detection. The results show an enahecement in fault and fracture detection. In the case of Marizeiro field, we needed to apply a noise attenuation procedure, which includes, Curvelet and a Structure-oriented filtering to improve data quality prior to MSA application.

\section{Introdução}

Um dado sísmico pode ser compreendido como uma representação de um modelo de sub-superfície terrestre, através de diversas sequências de interfaces, contínuas e descontínuas, muitas das quais associadas a estruturas geológicas, como falhas e fraturas. A qualidade do imageamento sísmico de tais estruturas é inversamente proporcional ao nível de ruído dos dados. Um dos objetivos do processamento sísmico é produzir imagens que honrem a geologia de sub-superfície e que possam ser interpretadas pelos exploracionistas. A otimização do imageamento e posicionamento de falhas e fraturas possui um papel fundamental na exploração de campos gás, cujos reservatórios possuem baixa permeabilidade. Tal otimização tem repercussões econômicas diretas em projetos exploratórios, no posicionamento e projeto de poços, no desenvolvimento de reservatórios, e planejamento de injeção e recuperação.

\section{O atributo MSA (Minimal Similarity Accumutaion)}

A coerência é uma medida de similaridade matemática que pode ser aplicada traço a traço no dado sísmico e serve como indicativo do grau de continuidade de eventos. Assume-se que corpos homogêneos e contínuos apresentem uma variação menor das amplitudes entre traços adjacentes do que corpos fraturados. A similaridade entre traços é assim um fator que pode ser utilizado para mapear regiões mais incoerentes no dado sísmico, onde há uma maior possibilidade da ocorrência de falhas e fraturas. Tais regiões, além apresentarem maior porosidade, podem, no caso da presença de falhas, conter rotas preferencias de migração de hidrocarbonetos. A identificação de tais áreas é fundamental, principalmente em se tratando de reservatórios de baixa permeabilidade.

Atributos de coerência vêm sendo utilizados há bastante tempo para detecção e mapeamento de falhas e fraturas. Gersztenkorn et al (1999), informam que os primeiros trabalhos aplicando medidas de coerência sísmica datam da década de 60, citando, como exemplo, o trabalho de Taner et al (1969), que utilizou a coerência para calcular os espectros de velocidade. O trabalho de Bahorich et al.(1995) é uma importante referência ao apresentar uma metodologia que adota uma combinação entre correlações cruzadas traço a traço (Inline e crossline), gerando o chamado cubo de coerência. Marfurt et. al. (1998) estenderam tal trabalho para um cenário multitraço, visando reduzir a influência do ruído nos resultados. Posteriormente, Gersztenkorn e Marfurt (1999) apresentaram um algoritmo de auto-estrutura da matriz de covariância, incorporando vários filtros e esquemas de interpolação para tratar ruídos e problemas de alias.

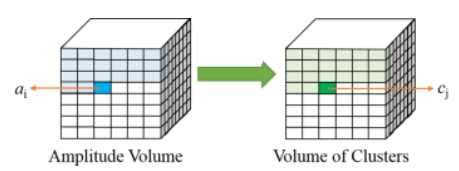

Figura 2 - Representação do agrupamento do volume amostrado (ai) no novo volume de classes (ci). O número de classes é definido pelo usuário (Figura gentilmente cedida por João Paulo Peçanha)

No mercado, há vários atributos de coerência, com diversos nomes dependendo do fornecedor, tais como Semblance, Similaridade, Caos, etc. Tais atributos diferem na preparação e no tratamento do dado, porém no interior dos algoritmos, em algum momento, há uma forma de determinação da correlação cruzada, buscando a determinação da coerência entre traços vizinhos.

O MSA é um atributo para identificação de descontinuidades, principalmente falhas e fraturas, disponível no $\mathrm{V} 302$ e que foi desenvolvido pela PUC- 
TecGraf. Ele também utiliza o cálculo da correlação cruzada e difere na forma como prepara o dado para tal cálculo. Ele é basicamente composto por quatro etapas1, que são as seguintes:

i) Amostragem do dado

a. Após a definição de uma janela pelo usuário, o algoritmo amostra o dado e gera um novo volume.

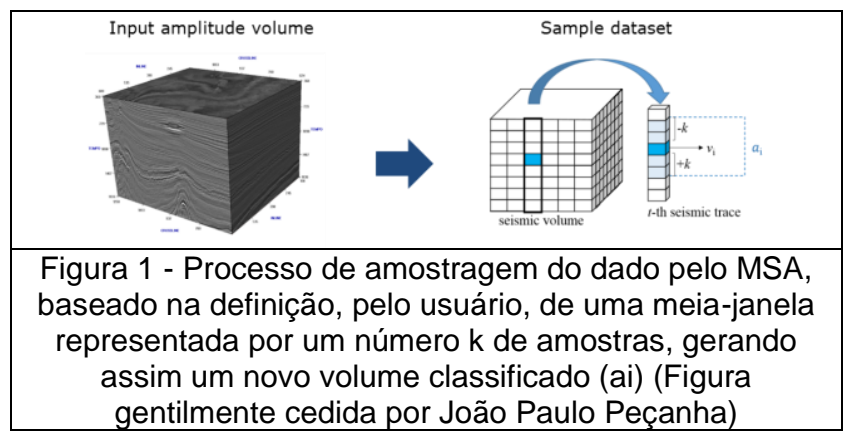

ii)

iii)

Redução de dimensionalidade

a. Esta etapa visa otimizar o processo de cálculo do atributo e não fazia parte da versão original do algoritmo.

Agrupamento de classes

a. Utilizando um esquema de aprendizado de máquina não supervisionado, o algoritmo classifica as amostras (ai) em determinado número de classes, definido pelo usuário, contendo elementos que compartilham características similares. Tais amostras são agora representadas no volume pela posição do grupo (ci) a que pertencem.

iv) Cálculo da correlação e saída do atributo.

Neste trabalho, para definição otimizada de descontinuidades estruturais sísmicas, apresenta-se um fluxo para atenuação de ruídos seguido pela aplicação de um atributo de coerência, denominado MSA (Figueiredo

et al,2014 \& Figueiredo et al.2016). Como précondicionamento dos dados, sugere-se a aplicação de uma sequência de filtros, Curvelet (Neelamani et al. 2008) e um filtro de suavização orientado por estrutura (FSOE) (Machado et al., 2015). Os resultados de aplicação de tal fluxo são ilustrados em dois volumes sísmicos empilhados, de Marizeiro e de Neuquén, ambos contendo gás em reservatórios de baixa permeabilidade.

\footnotetext{
${ }^{1}$ Para maiores detalhes sobre as etapas e toda a teoria do cálculo do MSA, ver Figueiredo et al. (2016)
}

\section{Campo de Marizeiro - Bacia Potiguar - Brasil}

A bacia Potiguar tem seu desenvolvimento iniciado no início do Cretáceo, resultando da abertura do Atlântico Sul (Françolin e Szatmari, 1987). A Bacia Potiguar ocupa uma área de aproximadamente $48.000 \mathrm{Km}^{2}$, onde 21.500 $\mathrm{km}^{2}$ em terra e $26.500 \mathrm{~km}^{2}$ em mar. Ela faz parte de um sistema de rift intracontinental que abriu de Sul para Norte no sul do Atlântico Sul e de Oeste para Este no ramo do Oceano Equatorial (Matos, 1992). O Campo de Marizeiro é um reservatório de baixa permeabilidade composto por arenitos e conglomerados com baixa permo-porosidade e pode ser definido como um reservatório do tipo tight gas sands. Devido a tais características, o mapeamento das zonas com maior densidade de fraturas é fundamental para embasar a locação de poços exploratórios e de desenvolvimento.

\section{Bloco Rio Neuquén - Bacia de Neuquén - Argentina}

O bloco Rio Neuquén está localizado a aproximadamente $30 \mathrm{~km}$ do NNE da cidade de Neuquén, bacia de Nequén, Argentinas, possuindo uma área de $397,59 \mathrm{~km}^{2}$. Reservatórios jurássicos de gás, com baixa peremabilidade, são encontrados na Formação Lajas (areias amalgamadas intercaladas por pelitos) e Punta Rosada (areias e argilas fluviais, deltaicas e estuarinas) que se configuram em plays de gás de centro de bacia. Para a produção destes reservatórios são fundamentais a compreensão da distribuição espacial e densidade das fraturas naturais existentes.

\section{O Pré-condicionamento de dados sísmicos}

A multiplicidade de um levantamento sísmico é um dos principais fatores que controlam a qualidade de dados sísmicos terrestres. Por multiplicidade, ou cobertura, entenda-se o número de vezes que determinado ponto em sub-superfície é amostrado em um levantamento sísmico. Quanto maior esse número, melhor será a razão sinal-ruído que está diretamente ligada à qualidade do dado. Algoritmos desenhados para detecção sísmica de fraturas são muito sensíveis à presença de ruídos. Como na sua maioria, tais algoritmos estão, basicamente, correlacionando, ou comparando, determinado traço com os seus vizinhos (Bahorich et al., 1995), a atenuação de ruídos é crítica para a sua performance.

Para atenuar a ruídos coerentes, no presente trabalho foi aplicado filtro do tipo Curvelet (Neelamani et al. 2008, op cit.) seguido por um filtro de suavização orientado por estrutura (FSOE) (Machado et al. 2015, op cit.), que busca melhorar a continuidade de refletores. Para destacar planos de falhas e possíveis fraturas, foi aplicada um processo de deconvolução iterativa - Iterdec (Rosa 2010), visando aumentar a resolução. Após o dado passar pelo fluxo da Figura 1, rodamos os algoritmos de MSA e Curvatura. 
Para analisarmos os resultados do fluxo de précondicionamento, apresentamos na Figura 2, seções sísmicas do dado original, painel superior e do dado após a filtragem, painel inferior. É possível perceber a redução de ruídos e a melhoria na visualização dos eventos. Após os filtros, aplicamos a deconvolução iterativa (Rosa,2010) e ilustramos o resultado na Figura 3, onde apresentamos no painel superior o dado após os filtros de Curvelet e FSOE e no painel inferior, a mesma seção após o Iterdec. Nos círculos amarelos, pode-se observar o ganho de qualidade e de resolução dado, originário do ganho no conteúdo de frequências devido à deconvolução. É importante destacar que os exemplos mostrados nas figuras 2 e 3, referem-se ao dado do campo de Marizeiro. Devido à alta qualidade do dado de Rio Neuquén, não foi necessária a aplicação do fluxo de pré-condicionamento antes do MSA.

Algoritmos de Curvatura podem ser utilizados para determinação de áreas fraturadas em exploração de reservatórios do tipo tight-gas de baixa permeabilidade (Chopra et al., 2007).

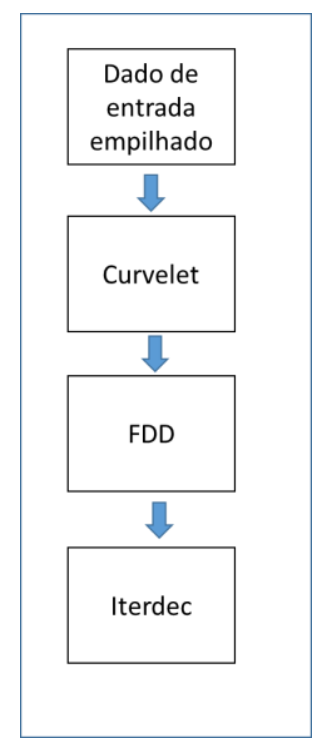

Figura 1. Esquema do fluxo de processamento utilizado para preparar o dado para a posterior aplicação do atributo para identificação de falhas e fraturas (MSA). O filtro Curvelet na atenuação de ruídos, o FDD na melhoria da continuidade dos refletores e a deconvolução iterativa (Iterdec) para o aumento da resolução.
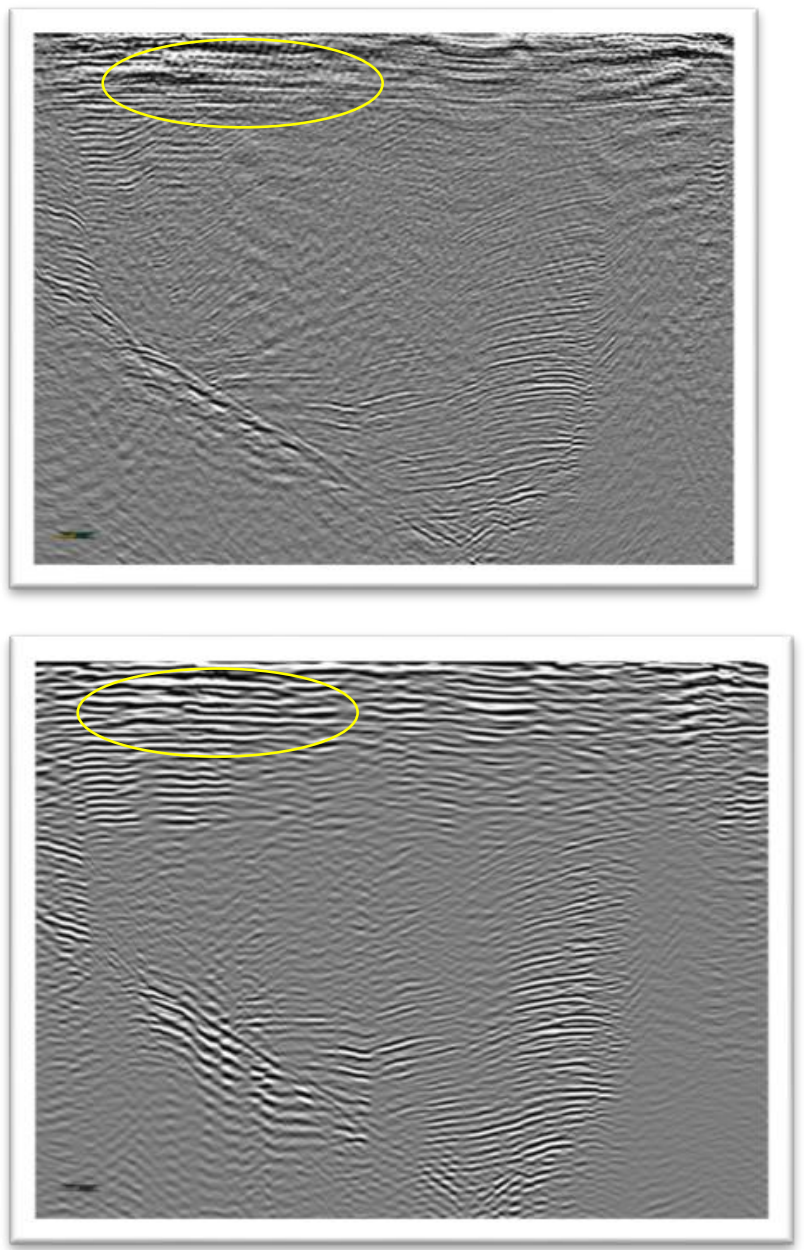

Figura 2. Seção do dado original (painel superior) e a mesma seção (painel inferior) após 0 précondicionamento com filtragem Curvelet e FDD no Campo de Marizeiro. Nota-se a melhora na qualidade da seção, após a aplicação dos filtros para atenuação dos ruídos (ver elipse em amarelo). 

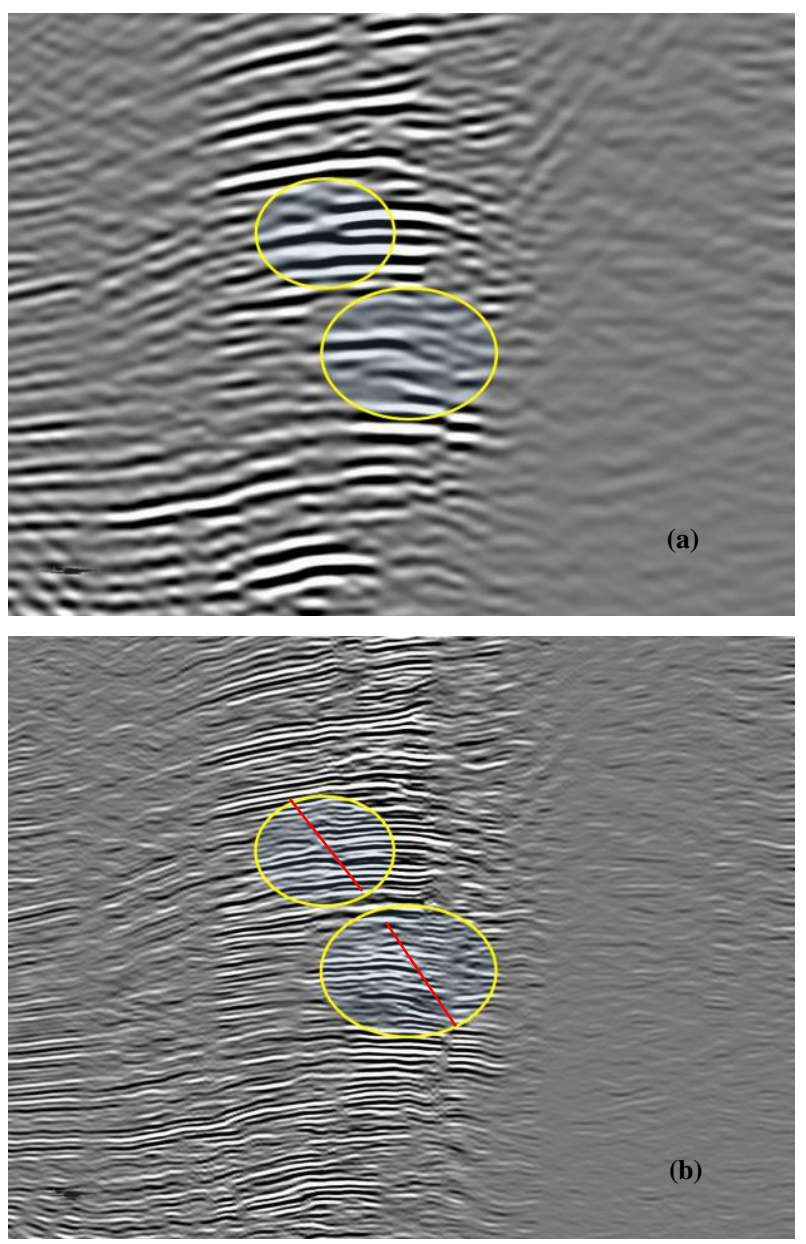

Figura 3. (a) Dado com filtragem Curvelet e FSOE. (b) Aplicação de filtros e Iterdec no Campo de Marizeiro. Nota-se o aumento da resolução e a melhor definição de possíveis planos de falhas em destaque (em vermelho) nos círculos amarelos.

\section{Resultados}

O procedimento descrito anteriormente, foi aplicado no volume empilhado de rio Neuquén. Para ilustrar, na Figura 4 (a) destaca-se um trecho de uma linha do dado original de entrada e em 4 (b), o dado após a aplicação do atributo MSA. Observa-se uma melhor visualização das estruturas de pequenas falhas, em destaque na elipse amarela. Estas estruturas são quase imperceptíveis no dado original podendo ser confundidas com ruído. A visualização de uma região mais falhada também no destaque na elipse em vermelho.

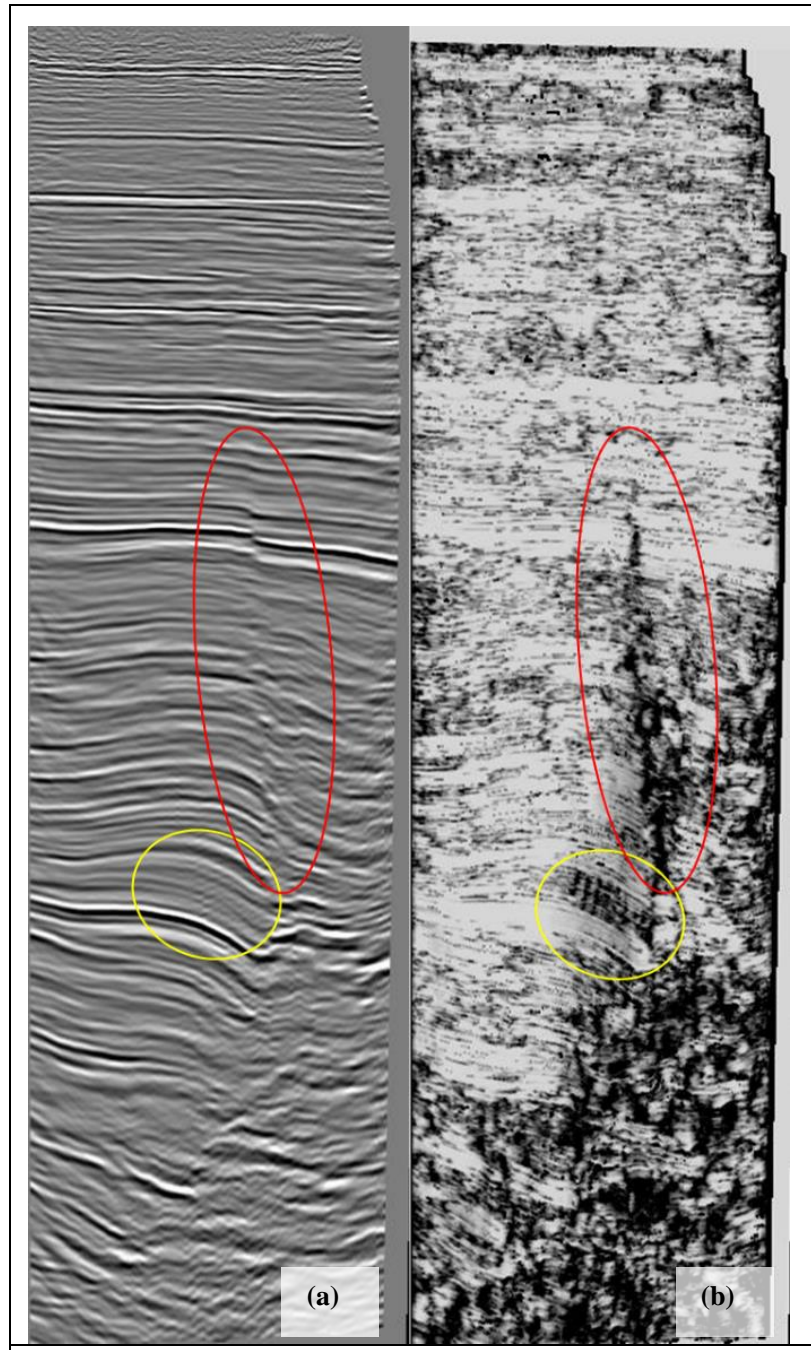

Figura 4. (a) Seção sísmica original do dado de Rio Neuquén, (b) mesma seção com aplicação do MSA. Observa-se uma melhor visualização de pequenas falhas, destaque no contorno em amarelo, no dado com MSA. Uma zona de falhas mais extensa, contorno em vermelho, pode ser melhor identificada no dado com MSA.

Devido à qualidade do dado de rio Neuquén, não foi necessário a aplicação do pré-condicionamento. No caso do volume do campo de Marizeiro, aplicamos o fluxo de filtros. As figuras 2 e 3 mostram o ganho em termos de qualidade do dado, atenuação de ruídos, e a melhora na resolução.

Na figura 6, apresenta-se uma fatia de tempo do volume sísmico de rio Neuquén, com a curvatura máxima sobre o MSA. Observa-se uma boa concordância entre ambos os atributos. A Curvatura máxima em vermelho pode indicar zonas mais curvadas e/ou fraturadas. 


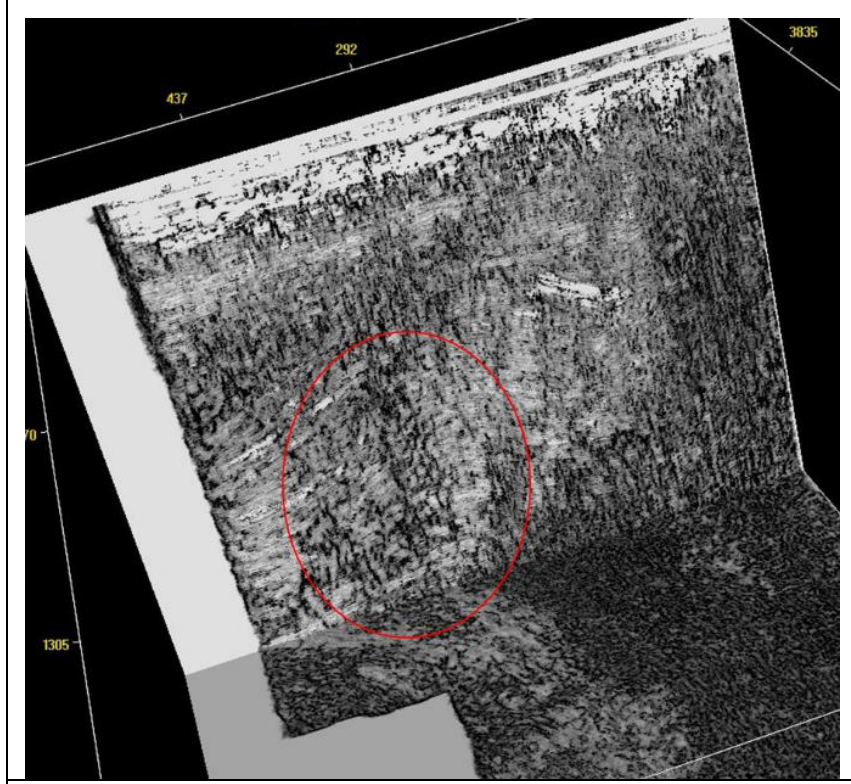

Figura 5. Volume de MSA do dado de Marizeiro após o pré-condicionamento. Fraturas são melhor identificadas com a aplicação do MSA (ver detalhe no contorno em vermelho).

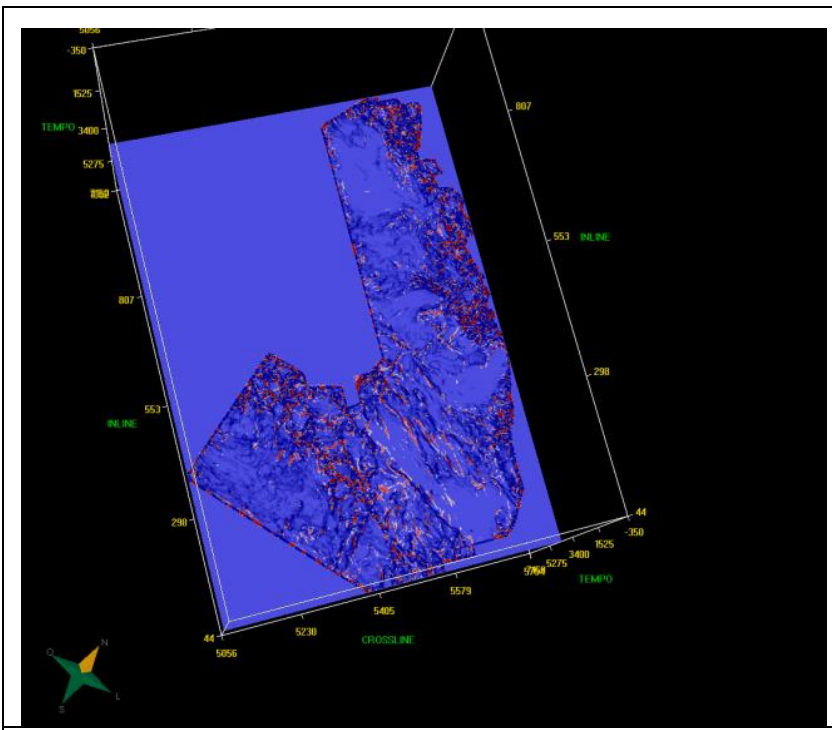

Figura 6. Fatia de tempo do dado de rio Neuquén mostrando os atributos Curvatura máxima (vermelho) e MSA. Observa-se uma concordância entre ambos, no mapeamento de lineamentos.
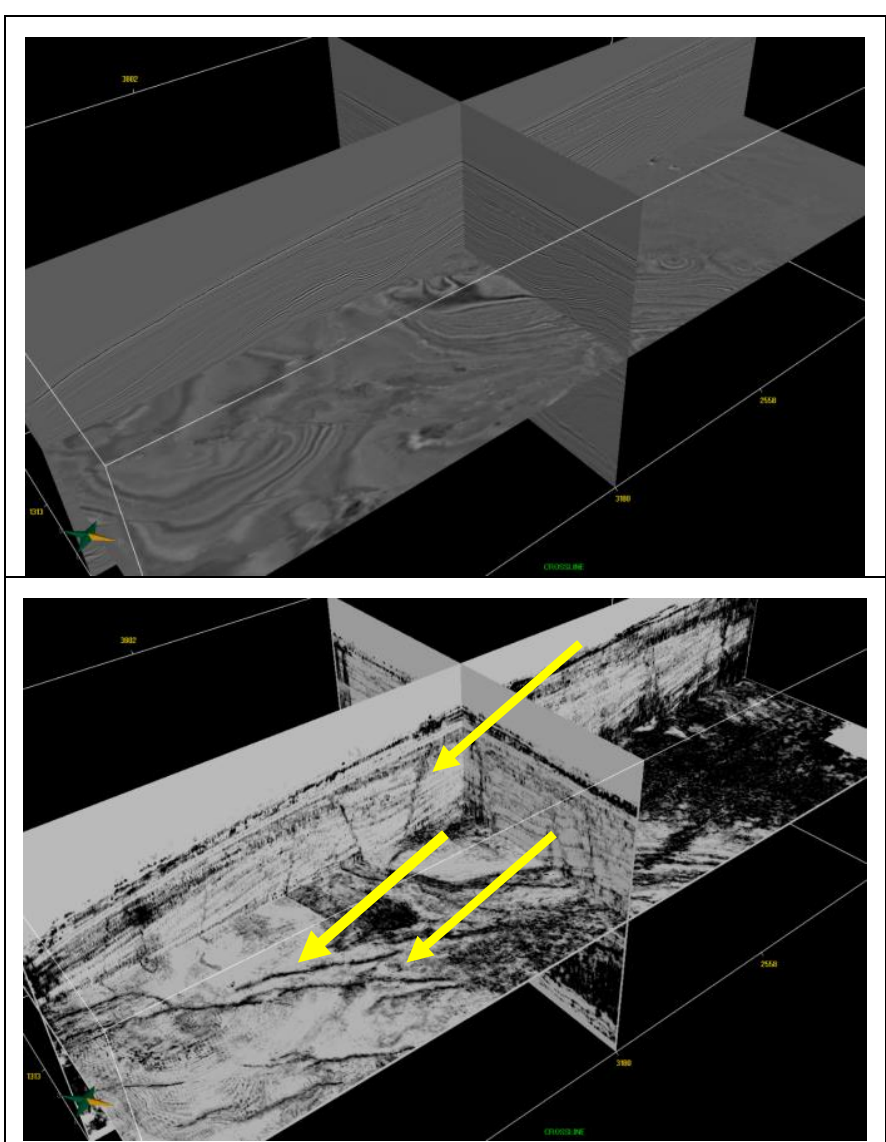

Figura 7 - No painel (a) dado sísmico original de um volume da área de Marlim Leste, no painel (b) o volume de MSA. Atentar para uma melhor visualização de possíveis falhas e lineamentos no volume de MSA (setas amarelas no painel (b)). 


\section{Comentários Finais}

$\mathrm{O}$ atributo MSA mostrou-se bastante efetivo melhorando a visualização de falhas como mostrado na figura 4 , Bacia Neuquén, na figura 5, Campo de Marizeiro, bacia Potiguar e principalmente no exemplo no Campo de Marlim Leste, da Bacia de Campos(figura 7). Os exemplos de Neuquén e Marlim Leste atestam o potencial do atributo na identificação e mapeamento de falhas e fraturas. É importante destacar que nos volumes de Neuquén e Marlim Leste não foi realizado nenhum tipo de filtragem pós-processamento. Os resultados em Marizeiro, após a aplicação do pré-condicionamento, foram bastante positivos, indicando regiões com maior densidade de fraturas. A melhor identificação de falhas permite a construção de um modelo estrutural mais realista, contribuindo para uma a redução do risco exploratório em bacias terrestres. O Iterdec (Rosa, 2010) propiciou uma melhoria significativa na resolução e se mostrou ferramenta importante para definição de planos de falhas e fraturas. A análise de qualidade do dado antes da aplicação do atributo, como forma de analisar a necessidade de aplicação de uma filtragem, ou précondicionamento, é fundamental no processo.

\section{Bibliografia}

Bahorich, M. e Farmer, S. (1995) " 3-D seismic discontinuity for faults and stratigraphic features: The coherence cube." The Leading Edge, 14(10), pp: 10531058.

Chopra, S. \& Marfurt, K.J., (2007) "Volumetric Curvatureattribute Application for Detection of Fracture Lineaments and Their Calibration", Geohorizons, Dezembro 2007/27, Society of Petroleum Geophysicists.

Figueiredo, A.M., Faustino, G.M., Peçanha,J.P., Silva, P.M. e Gattass, M. (2014) "Minimal Similarity Accumulation Attribute for Fault Enhancement", 84 $4^{\text {th }}$ SEG Annual Meeting - Denver, EUA.

Figueiredo, A.M., Peçanha,J.P., Faustino, G.M., Silva, P.M. e Gattass, M. (2016) "High Quality Horizon MappingUsing Clustering Algorithms" - 78th EAGE Conference and Exhibition, Viena, Austria

Françolin, J.B.L., Szatmari, P. (1987) Mecanismo de rifteamento da porção oriental da margem norte brasileira. Revista Brasileira de Geociências, v. 17, p.196207.

Gersztenkorn, A. e Marfurt, K.J. (1999). "Eigenstructurebased coherence computations as an aid to 3-D structural and stratigraphic mapping." Geophysics, Vol. 64, № 5 , pp:1468-1479.

MATOS, R.M.D. (1992) Deep seismic profiling, basin geometry and tectonic evolution of intracontinental rift basins in Brazil. U.S.A.: Cornel University (Tese de Doutorado).

Neelamani,R. , Baumstein, A.I., Gillard,D.G. Hadidi,M. T. e Soroka, W.L. (2008). "Coherent and random noise attenuation using the curvelet transform." The Leading Edge,27(2),240-248. 